\title{
Measures of Durability of Resistance
}

\author{
F. van den Bosch and C. A. Gilligan
}

First author: Biomathematics, Rothamsted Research, Harpenden, Herts AL5 2JQ, UK; and second author: Department of Plant Sciences, University of Cambridge, Downing Street, Cambridge CB2 3EA, UK.

Accepted for publication 27 December 2002.

\begin{abstract}
van den Bosch, F., and Gilligan, C. A. 2003. Measures of durability of resistance. Phytopathology 93:616-625.

Conventional models for the durability of resistant cultivars focus on the dynamics of the frequency of resistance genes. This leads to a definition of the durability of resistance as the time from introduction of the cultivar to the time when the frequency of the virulence gene reaches a preset threshold. It is questionable whether this is the most appropriate way to measure durability. Here we use a simple epidemiological model to link population dynamics and population genetics to compare three measures of durability: (i) the expected time until invasion of the virulent genotype, by mutation or immigration, and subsequent establishment of a

population $\left(T_{\text {invasion }}\right)$; (ii) the virulence frequency related measure of the time for the virulent genotype to take-over the pathogen population $\left(T_{\text {take-over }}\right)$; and (iii) the additional yield, measured by the additional number of uninfected host growth days $\left(T_{\text {additional }}\right)$. Specifically, we show how the measures of durability are affected by deployment and epidemiological parameters. We use a combination of numerical solution and analytical approximation of a model for the population dynamics of avirulent and virulent genotypes of a pathogen growing in dynamically changing populations of resistant and susceptible cultivars. The three measures of durability are compared. Some consequences of the results for durable resistance in multilines and mixtures and the regional deployment of resistant cultivars are discussed.
\end{abstract}

The objectives of this paper are twofold. Firstly, three measures of durability of a cultivar resistant to a plant pathogen are compared, two of which are new to the study of disease resistance in crops. Secondly, the dependence of these measures on the cropping ratio of the resistant cultivar and on epidemiological parameters is studied. In this introduction, we argue that the measure of durability conventionally used by plant pathologists only represents one aspect of durability. Next, two new measures of durability are introduced describing other aspects of durability. Finally, the approach taken in this paper is described.

The deployment of resistant cultivars is a widely used method for pest and disease management. Limited durability of the resistance, however, remains a major problem in the deployment of resistant cultivars. The selection pressure on the parasite population selects for virulent forms rendering the resistant cultivar ineffective. The period between the release of a resistant cultivar and the end of its usefulness for disease management differ markedly among host-pathogen systems. Some resistance genes have remained effective for a long time. Notable among these is resistance to cabbage yellows caused by Fusarium oxysporum f. sp. conglutinans, which has lasted for more than 90 years (37), and resistance to leaf rust, Puccinia triticina, conferred by Lr34, which has lasted for 30 years (19). Others are remarkably ephemeral, for example, $\mathrm{Yr} 17$ for the control of yellow rust on wheat was rapidly overcome in two to three seasons by virulent isolates of $P$. striiformis f. sp. tritici in the U.K. followed by Denmark, France, and Germany (1). The durability of various rice blast resistance genes is often less than 3 years $(18,40)$. Various deployment strategies have been proposed to increase the durability of resistance genes. These include restriction of the area sown to resistant cultivars, rotational use of cultivars with different resistance genes, pyramiding of resistance genes within one cultivar,

Corresponding author: F. van den Bosch

E-mail address: frank.vandenbosch@bbsrc.ac.uk

Publication no. P-2003-0314-02R

(C) 2003 The American Phytopathological Society and integration of resistant cultivars with fungicidal treatments. In this paper, we focus on controlling the area sown to resistant crops, termed the cropping ratio, $\phi$.

The durability of resistance has attracted empirical and theoretical attention to gain insight into mechanisms that favor prolonged resistance (recent reviews of Vera Cruz et al. [37] and Parlevliet [32]). Johnson (14-16) formalized the empirical identification of sources of durable resistance, by monitoring persistence under wide-scale cultivation, and showed how to use these in selection programs $(13,17)$. Following early work by Vanderplank (36) and Leonard (22), an increasing number of models to study virulence dynamics in plant pathogens have been published $(3,12,20,23$, 25,27).

A common characteristic of these models is that they consider the dynamics of the relative frequencies, rather than densities, of genes and genotypes in the pathogen population. This approach stems from the modeling approaches developed in theoretical population genetics (8). Correspondingly, most experimental studies also consider the frequency of virulence genes $(4,5,28,29)$. It follows from these studies that the obvious way to measure durability is the time-span from introduction of the resistant cultivar to the time when frequency of the virulence gene reaches a preset threshold, above which resistance is considered broken down. This definition of durability is, however, restrictive when considering the deployment of resistance genes to maximize the potential advantage for disease control. When the cropping ratio of the resistant cultivar is small, the durability, as defined previously, is large. The contribution of the cultivar to the total crop yield however is small. Conversely, when the cropping ratio is large, the contribution of the resistant cultivar to yield is large but durability is small. Durability defined in this way, therefore, does not reflect the use of the resistant cultivar to increase yield, which is the ultimate practical goal of using resistant cultivars.

Another problem is that the models usually assume the virulent genotype is present in the genetic background of the pathogen population, i.e., that there is preexisting virulence (36). This results in an increase of the virulent genotype from the moment of release of the resistant cultivar. It fails, however, to provide a 
measure for durability that takes account of the time for the virulent genotype to enter the system (through mutation or immigration) and establish a population when the virulent genotype is absent from the region of deployment. Extension of the concept of durability to consider invasion and yield requires a population dynamic, as opposed to a population genetic, model formulation.

New measures of durability. In this paper, we study three definitions of durability and their relationships with cropping ratios and epidemiological parameters. First, we assume that the virulent pathogen genotype is absent from the population. Durability can then be defined as the time-span from the release of the resistant cultivar until the virulent genotype appears in the population through mutation or immigration and subsequently establishes a population. The process of "appearance" and subsequent "establishment" together constitute an "invasion." This measure is the expected time until virulence invasion, $T_{\text {invasion }}$.

For comparison, we study the traditionally used virulence frequency related measure of durability. This measure is defined as the time-span from the release of the resistant cultivar to the time when the frequency of the virulent pathogen genotype reaches a preset (in our case 90\%) level, given that the virulent genotype is present in the genetic background of the pathogen population. This measure is the time until the virulent genotype takes over the pathogen population, $T_{\text {take-over }}$.

Assuming that yield is largely determined by the number of days during the growing season that a plant is uninfected, we can also define durability as the additional number of uninfected host growth days added due to the use of the resistant cultivar. This measure is the added number of uninfected host growth days, $T_{\text {additional. }}$ A comparable measure was first used by Bonhoeffer et al. (2) in models for antibiotic resistance.

The approach. We derive a simple model. The model ignores spatial structure. Further, we assume that the pathogen reproduces asexually and that there are overlapping generations of crop and pathogen. In parts of the paper where mutations are considered, it is assumed that avirulent pathogens mutate into virulent pathogens. This assumption corresponds most closely to a single gene mutation. Furthermore, we will often assume that the cultivar and the pathogen strain (or strains) present in the system are in steady state at the moment of release of the resistant cultivar.

Specifically, we study how the measures of durability are affected by (i) deployment parameters represented by the cropping ratio of the resistant cultivar and (ii) epidemiological parameters for transmission, migration, and mutation of pathogen genotypes.

The approach, using a simple model, allows us to derive a set of relations between cropping ratio, epidemic parameters, and durability that will serve as baseline results in future model expansions. In the discussion, we describe how these simplifying assumptions will be relaxed in future research.

The questions are addressed by a combination of numerical solution and analytical approximation of a model for the dynamics of avirulent and virulent genotypes of a pathogen growing in dynamically changing populations of resistant and susceptible cultivars.

\section{THEORY AND APPROACHES}

The model. Consider a pathosystem open to pathogen immigration with a susceptible and a resistant cultivar infected by an avirulent and a virulent pathogen genotype. The density of uninfected susceptible cultivar, $H_{S}(t)$, and uninfected resistant cultivar, $H_{R}(t)$, increase in time due to planting and decrease due to harvesting. Both the virulent and the avirulent pathogen genotypes can infect the susceptible cultivar, but only the virulent genotype can infect the resistant cultivar. The virulent, $V(t)$, and the avirulent, $A(t)$, pathogen genotypes increase due to infection and a small rate of immigration and decrease due to death. This leads to the system of four differential equations: $d H_{S}(t) / d t=(\text { planting })_{S}-(\text { harvesting })_{S}-(V \text { infection })_{S}-(A \text { infection })_{S}$

$$
\begin{aligned}
d H_{R}(t) / d t & =(\text { planting })_{R}-(\text { harvesting })_{R}-(V \text { infection })_{R} \\
d A(t) / d t & =\left(\text { infectious } H_{S}\right)_{A}-(\text { death })_{A}+(\text { immigration })_{A}
\end{aligned}
$$

$d V(t) / d t=\left(\text { infectious } H_{S}\right)_{V}-\left(\text { infectious } H_{R}\right)_{V}-(\text { death })_{V}+(\text { immigration })_{V}$

The amounts of plant tissue infected by the avirulent or the virulent strains are used as measures of pathogen density so we do not explicitly keep track of infected plant density. We assume a constant total planting rate, $\sigma$. A fraction, $\phi$, of the planted crop is the resistant cultivar and a fraction $(1-\phi)$ is the susceptible cultivar. This leads to (planting $)_{S}=(1-\phi) \sigma$ and (planting $)_{R}=\phi \sigma$. The period between planting and harvesting has a length of $1 / \omega$ time units which results in the crop harvest terms (harvest) $)_{S}=\omega H_{S}(t)$ and (harvest) $)_{R}=\omega H_{R}(t)$. The net effect of sowing and harvest is a monomolecular approach to an asymptotic limit in the increase of host tissue over time in the absence of disease. The limit for each host type is determined by the cropping ratio, thus $d H_{S} / d t=$ $\omega\left[\sigma(1-\phi) / \omega-H_{S}\right]$ and $d H_{R} / d t=\omega\left(\sigma \phi / \omega-H_{R}\right)$.

The pathogen produces $\xi$ spores per unit time per unit area. The probability that a spore lands on an uninfected host and causes an infection is assumed to be linearly related to uninfected host density, with proportionality constant $\alpha$. The infection rates of uninfected hosts can thus be written as $(V \text { infection })_{S}=\beta H_{S}(t) V(t)$, $(V \text { infection })_{R}=\beta H_{R}(t) V(t)$, and $(A \text { infection })_{S}=\beta H_{S}(t) A(t)$, where $\beta=\xi \alpha$ is the infection rate.

Each infection results in $g$ spore-producing pathogen units, resulting in the terms (infectious $\left.H_{S}\right)_{A}=\beta g H_{S}(t) A(t)$, (infectious $\left.H_{S}\right)_{V}=\beta g H_{S}(t) V(t)$, and (infectious $\left.H_{R}\right)_{V}=\beta g H_{R}(t) V(t)$. Pathogens have a constant probability per unit time to die, which gives $(\text { death })_{A}=\mu A(t)$ and $(\text { death })_{V}=\mu V(t)$. We assume a small constant rate of pathogen immigration, thus, (immigration) $)_{A}=\delta_{1}$ and $(\text { immigration })_{V}=\delta_{2}$.

The model equations thus read

$$
\begin{gathered}
d H_{S}(t) / d t=(1-\phi) \sigma-\omega H_{S}(t)-\beta H_{S}(t) A(t)-\beta H_{S}(t) V(t) \\
d H_{R}(t) / d t=\phi \sigma-\omega H_{R}(t)-\beta H_{R}(t) V(t) \\
d A(t) / d t=\beta g H_{S}(t) A(t)-\mu A(t)+\delta_{1} \\
d V(t) / d t=\beta g H_{S}(t) V(t)+\beta g H_{R}(t) V(t)-\mu V(t)+\delta_{2}
\end{gathered}
$$

TABLE 1. Variables and parameters used in the model and their interpretation and dimension

\begin{tabular}{llc}
\hline Variables & Interpretation & Dimension \\
\hline$H_{\mathrm{S}}$ & Density of susceptible cultivar & $L^{-2}$ \\
$H_{\mathrm{R}}$ & Density of resistant cultivar & $L^{-2}$ \\
$A$ & Density of avirulent pathogen & $L^{-2}$ \\
$V$ & Density of virulent pathogen & $L^{-2}$ \\
Parameters & & \\
$\beta$ & Infection rate & $t^{-1}$ \\
$\delta_{1}$ & Immigration rate of avirulent pathogen & $t^{-1}$ \\
$\delta_{2}$ & Immigration rate of virulent pathogen & $t^{-1}$ \\
$\phi$ & Cropping ratio & 1 \\
$g$ & Number of spore producing pathogen units & $\#$ \\
& per infection & $L^{2}$ \\
$\sigma$ & Area of the pathosystem & $t^{-1}$ \\
$\mu$ & Planting rate & $t^{-1}$ \\
$\omega$ & $1 / \mu$ is the pathogen average life span & $t^{-1}$ \\
\hline a $L$ is length and $t$ is time &
\end{tabular}

${ }^{a} L$ is length and $t$ is time. 
Definitions and dimensions of the variables and parameters are summarized in Table 1.

Model dynamics. The dynamical behavior of the model in equation 2 is not the subject of this paper. To put the calculation of the three measures of durability into perspective, however, we require some information on the dynamics for seven scenarios: for presence and absence of the resistant cultivar with and without immigration of the pathogen. These are summarized in Table 2. The virulent migrants are assumed to be much rarer than avirulent migrants either because the source of the virulent migrants is much farther away or because both types of migrants come from a common source in which the virulent genotype is rare. We therefore assume throughout the paper that $\delta_{2}$ is very small. In situations in which this assumption does not hold and $\delta_{2}$ is large, durability will be close to zero and investigating the effects of epidemic processes on durability is no longer relevant.
Calculating the measures of durability of resistance. In this section, we describe the numerical methods used to calculate the three measures of durability. Numerical solutions are used because analytical solution of the general model (equation 2) is not possible. In the results section, we derive several approximations analytically, most of them relying on the assumption that immigration is very small or entirely absent from the system.

Time until the virulent pathogen genotype invades, $T_{\text {invasion. }}$ Consider a system in which the resistant cultivar is not yet in use $(\phi=$ 0 ) and the virulent pathogen genotype is absent from the system. Uninfected susceptible crop and avirulent genotype are in steady state (Table 2). The introduction of the resistant cultivar is mimicked by changing the planting ratio, $\phi$, from zero to the appropriate value at time $(t)=0$. The density of the resistant crop will in the course of time increase from zero to its final level, $\bar{H}_{R}=\phi \sigma / \omega$. Because the avirulent genotype cannot infect the re-

TABLE 2. Summary of the dynamics of model equations 2 for four scenarios involving presence or absence of the resistant cultivar with and without immigration of pathogens ${ }^{\mathrm{a}}$

\begin{tabular}{|c|c|c|c|}
\hline Resistant cultivar & $\begin{array}{l}\text { Immigration/ } \\
\text { no immigration }\end{array}$ & Summary & \\
\hline $\begin{array}{l}\text { Resistant cultivar } \\
\text { absent } \phi=0\end{array}$ & $\begin{array}{l}\text { No immigration } \\
\delta_{1}=0, \delta_{2}=0\end{array}$ & $\begin{array}{l}\text { Both pathogen } \\
\text { strains present }\end{array}$ & $\begin{array}{l}\text { Given both genotypes are present at } t=0, A \text { and } V \text { coexist for all time if } \\
\qquad \frac{\beta g}{\mu} \frac{\sigma}{\omega}>1 \text {, } \\
\text { i.e., pathogen net-reproductive number }>1 . A(t) /[V(t)+A(t)]=A(0) /[V(0)+A(0)] \text {. Internal steady } \\
\text { state is stable: }\end{array}$ \\
\hline & & & $\bar{H}_{S}=\frac{\mu}{\beta g}, \quad \bar{A}+\bar{V}=\frac{\sigma g}{\mu}-\frac{\omega}{\beta}, \quad \frac{\bar{A}}{\bar{V}+\bar{A}}=\frac{A(0)}{V(0)+A(0)}$ \\
\hline
\end{tabular}

Immigration Both pathogen

$\delta_{1}>0, \delta_{2}>0 \quad$ strains present

Resistant cultivar present $\phi>0$

No immigration Both pathogen $\delta_{1}=0, \delta_{2}=0 \quad$ genotypes absent

Avirulent genotype present, virulent genotype absent

Both pathogen genotypes present

Immigration $\delta_{1}>0, \delta_{2}=0$

Immigration $\delta_{1}>0, \delta_{2}>0$ present, virulent genotype absent
Both pathogen genotypes present

If the virulent pathogen strain is absent from the system the equations apply with $V(t)=V(0)=0$.

$A$ and $V$ coexist and for $t \rightarrow \infty, A / V \rightarrow \delta_{1} / \delta_{2}$. Internal steady state is stable:

$$
\begin{gathered}
\bar{H}_{S} \frac{\mu(\bar{A}+\bar{V})-\delta_{1}-\delta_{2}}{\beta g(\bar{A}+\bar{H})}, \quad \frac{\bar{V}}{\bar{A}}=\frac{\delta_{2}}{\delta_{1}}, \quad \bar{A}+\bar{H}=\frac{1}{2}\left(\Theta+\sqrt{\Theta^{2}+4 \Omega}\right), \\
\Theta=\frac{\sigma g}{\mu}-\frac{\omega}{\beta}-\frac{\delta_{1}+\delta_{2}}{\mu}, \quad \Omega=\frac{\omega}{\beta} \frac{\delta_{1}+\delta_{2}}{\mu}
\end{gathered}
$$

Resistant and susceptible cultivar both present at all time. Cultivar densities approach

$$
\bar{H}_{S}=\frac{(1-\phi) \sigma}{\omega}, \quad \bar{H}_{R}=\frac{\phi \sigma}{\omega}
$$

Coexistence of resistant and susceptible cultivar and the avirulent pathogen genotype if

$$
\frac{\beta g}{\mu} \frac{(1-\phi) \sigma}{\omega}>1
$$

i.e., avirulent pathogen strain net-reproductive number $>1$. The internal steady state is stable:

$$
\bar{H}_{S}=\frac{\mu}{\beta g}, \quad \bar{H}_{R}=\frac{\phi \sigma}{\omega}, \quad \bar{A}=\frac{(1-\phi) \sigma g}{\mu}-\frac{\omega}{\beta}
$$

Coexistence of resistant and susceptible cultivar and the virulent pathogen strain if

$$
\frac{\beta g}{\mu} \frac{\sigma}{\omega}>1
$$

i.e., virulent pathogen strain net-reproductive number $>1$. The steady state is stable and the avirulent genotype goes extinct:

$$
\bar{H}_{S}=\frac{(1-\phi) \mu}{\beta g}, \quad \bar{H}_{R}=\frac{\phi \mu}{\beta g}, \quad \bar{V}=\frac{\sigma g}{\mu}-\frac{\omega}{\beta}, \quad \bar{A}=0
$$

Avirulent genotype Resistant cultivar dynamics independent of the dynamics of susceptible cultivar and avirulent pathogen strain. Avirulent pathogen strain exists irrespective of parameter values. All variables converge to steady-state value

$$
\begin{gathered}
\bar{H}_{R}=\frac{\phi \sigma}{\omega}, \quad \bar{A}=\frac{1}{2}\left(\Theta+\sqrt{\Theta^{2}+4 \Omega}\right), \quad \Theta=-\frac{g}{\mu}\left[(1-\phi) \sigma-\frac{\delta_{1}}{g}\right]+\frac{\omega}{\beta}, \quad \Omega=\frac{\delta_{1} \omega}{\beta \mu}, \\
\bar{H}_{S}=\frac{1}{\omega}\left[(1-\phi) \sigma-\frac{\delta_{1}}{g}\right]-\frac{\mu}{\omega g} \bar{A}
\end{gathered}
$$

Coexistence of susceptible and resistant cultivar with both avirulent and virulent pathogen genotype. The steady state densities can only be solved numerically. The avirulent pathogen genotype does not die out only due to continuous immigration, steady state densities of $A$ are very small since immigration is small. Internal steady state is stable.

\footnotetext{
a When a cultivar or pathogen genotype is absent, the equation describing its dynamics and all terms in the other equations involving its density are deleted from model equations 2 . Entries in the table describe the dynamics of this reduced system.
} 
sistant cultivar, its resource, the susceptible cultivar decreases and subsequently the density of the avirulent genotype also decreases. In the long term, it converges to a new steady state. After a transient period, the density of the uninfected susceptible crop settles at the same steady-state density before the introduction of the resistant cultivar. This situation is shown in Figure 1A.

In this system, avirulent genotypes mutate at a low rate into virulent genotypes and virulent genotypes immigrate at a low rate into the system. We assume the immigration rate of the virulent pathogen, $\delta_{2}$, to be so small that immigration is a chance process. How long will it take before the virulent genotype invades the system? Two processes determine the time until invasion of the virulent genotype, $T_{\text {invasion }}$. First, an avirulent genotype has to mutate into a virulent genotype or a virulent genotype has to immigrate; this process is termed appearance. Secondly, after a mutation has occurred or an immigrant has arrived, there is one virulent genotype individual. This pathogen has to build up an initial population; this process is termed establishment (38). At such low densities, the pathogen is highly sensitive to random (demographic) fluctuations. Many of the appearances go extinct before the initial population has established and will therefore never be of influence or be detected in the field. We thus define the virulent strain to have invaded after it has appeared and subsequently established a population.

Appearance is calculated as follows. Each avirulent genotype has a probability $\varepsilon$ per unit time to mutate into a virulent genotype. The number of mutations into a virulent genotype thus equals the product of the mutation rate and the number of the avirulent genotype individuals, $\varepsilon O A(t)$, where $O$ is the area of the system. Before the virulent genotype has invaded, the total number of virulent genotypes entering the system per unit time, $K_{1}$, is

$$
K_{1}=\varepsilon O A(t)+\delta_{2} O
$$

Establishment is calculated as follows. Various authors $(6,24,35)$ have shown that the probability, $K_{2}$, of not going extinct due to random fluctuations is given by

$$
K_{2}=1-1 / R_{0}
$$

where $R_{0}$ is the basic reproductive number. In our case, the basic reproductive number of the virulent genotype has to be calculated with the system of susceptible cultivar, resistant cultivar, and avirulent genotype (Fig. 1A) as the background environment of the virulent genotype, denoted here by $R_{0}^{\prime}$.

A virulent genotype can infect both the susceptible and the resistant cultivar. The number of new infections in the susceptible cultivar per unit time is $\beta g H_{S}(t)$ and in the resistant cultivar is $\beta g H_{R}(t)$. The pathogen's average life span is $1 / \mu$. Combining these, we find the basic reproductive number

$$
R_{0}^{\prime}=\frac{\beta g H_{S}(t)+\beta g H_{R}(t)}{\mu}
$$

The probability per unit time that the virulent genotype appears through a mutation or immigration and establishes, $\rho(t)$, is the product of the number of mutations and immigrations per unit time, $\varepsilon O A(t)+\delta_{2} O$, and the probability it will establish, $K_{2}$. Given $\rho(t)$, the probability density of the waiting time until invasion of the virulent genotype, $f(t)$, can be calculated. Once this density is known, the expected time until invasion, $T_{\text {invasion, }}$, is found from

$$
T_{\text {invasion }}=\int_{0}^{\infty} t f(t) d t
$$

The cumulative probability density, $F(t)$, of the probability density, $f(t)$, can be interpreted as the probability that the virulent genotype has established at time $t$. We can derive a differential equation that describes the rate of change of $F$ with time. The probability that the virulent genotype establishes in a small time interval from time $t$ to $t+\Delta t$ equals $F(t+\Delta t)-F(t)$. It is also equal to the probability that the virulent genotype has not yet established at time $t, 1-F(t)$, multiplied by the probability that it will become established in this time interval. For a short period of time, $\Delta t$, the latter probability can be approximated with $\rho(t) \Delta t$. We thus have

$$
F(t+\Delta t)-F(t) \approx \rho(t) \Delta t[1-F(t)]
$$

Dividing both sides by $\Delta t$ and taking the limit of $\Delta t \rightarrow 0$, we arrive at the differential equation

$$
d F(t) / d t=\rho(t)[1-F(t)]
$$

Given a solution of model equations 2 , it is possible to calculate $\rho(t)$, which in its turn makes it possible to solve differential equation 8 and, hence, to obtain the expected waiting time until invasion of the virulent genotype.
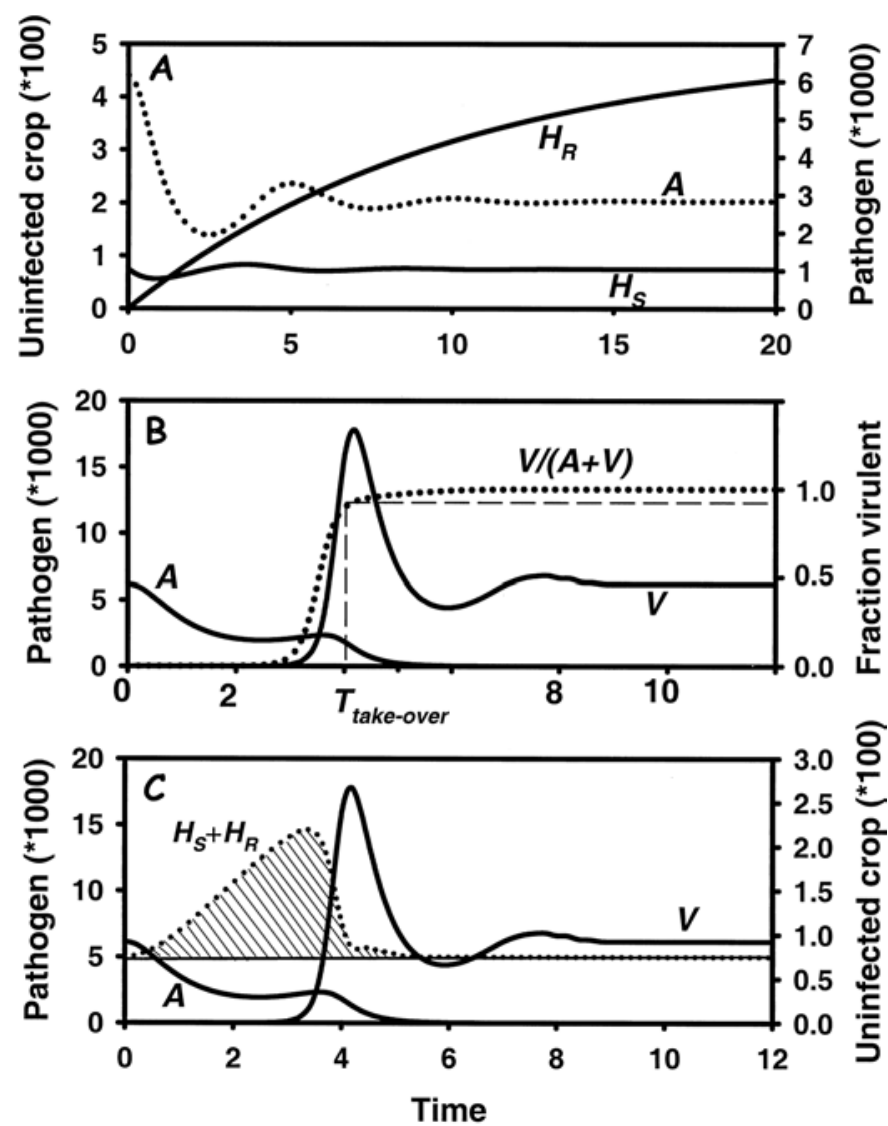

Fig. 1. Dynamics of model equation 2. Parameter values set at $\beta=2 \times 10^{-4}$, $g=200, \mu=3, \omega=0.1, \sigma=100, \delta_{1}=20$, and $\delta_{2}=0.02$, unless stated otherwise. A, Dynamics of equation 2 when the virulent pathogen genotype is absent from the system at time $(t)=0$, and when the resistant cultivar is released and no immigration of the virulent pathogen takes place and no avirulent pathogens mutate into virulent pathogens. Compare this graph with equilibrium densities in Table 2, fourth row. B, Dynamics of the model when at $t=0$ the virulent pathogen genotype is present in very low densities and the resistant cultivar is released and deployed from $t=0$. Dotted line is the fraction of the pathogen population virulent. The $T_{\text {take-over }}$ on the $x$ axis shows the definition of the time until the virulent genotype comprises $90 \%$ of the pathogen population. This quantity is used in Figure 3. Compare this graph with Table 2, sixth row. $\mathbf{C}$, Same simulation as middle graph but also shows the total density of uninfected crop, $H_{S}+H_{R}$. The hatched area shows the definition of $T_{\text {additional }}$, the total number of additional uninfected crop growth days due to the release and deployment of the resistant cultivar. This quantity is used in Figure 4. Compare this graph with Table 2, sixth row. 
For later use, we note that equation 8 can be solved, and after differentiating with respect to time, we obtain the probability density of the waiting time until invasion of the virulent genotype

$$
f(t)=\rho(t) \exp \left[\int_{0}^{t} \rho(x) d x\right]
$$

Time until the virulent genotype takes over, $T_{\text {take-over }}$ Consider a system in which the resistant cultivar is not in use $(\phi=0)$. Uninfected susceptible crop and pathogen are in steady state. In this system, the resistant cultivar is introduced. The virulent genotype is already present at low densities in the genetic background of the pathogen population. It is at a selective advantage once the resistant cultivar is introduced and will start to increase. In the

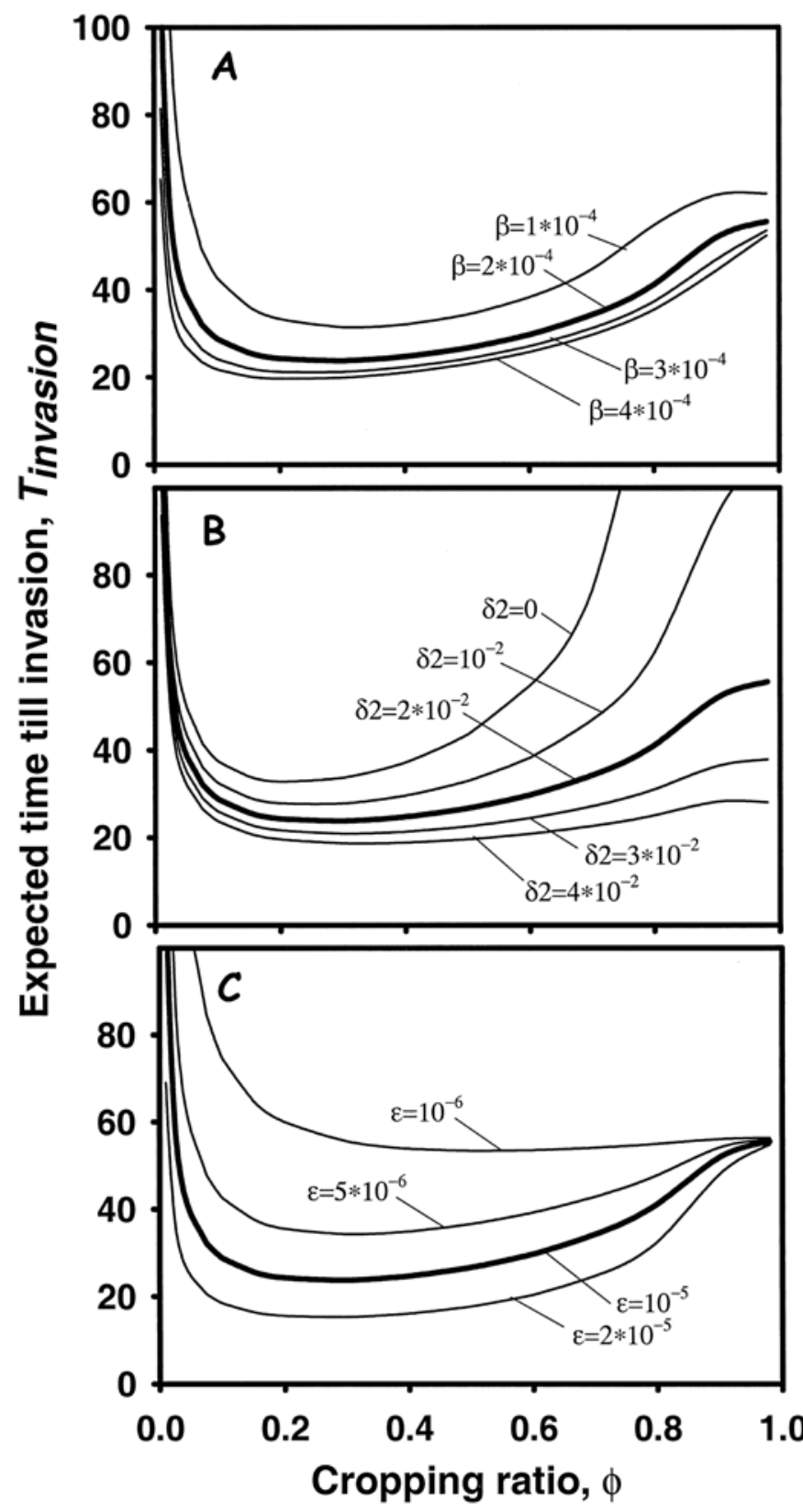

Fig. 2. Expected time until the virulent pathogen genotype invades, $T_{\text {invasion, }}$, as a function of the fraction of resistant cultivar planted (cropping ratio, $\phi$ ). $A$, The effect of infection rate, $\beta$. B, The effect of immigration rate of the virulent pathogen genotype, $\delta_{2}$. C, The effect of mutation rate, $\varepsilon$. Bold lines indicate the default parameter set at $\beta=2 \times 10^{-4}, g=200, \mu=3, \omega=0.1, \sigma=$ $100, \delta_{1}=20, \delta_{2}=0.02, \varepsilon=10^{-5}$, and $O=1$. long term, the system stabilizes at a new steady state (Table 2, bottom row). Figure 1B shows the dynamics of both pathogen genotypes together with the dynamics of the frequency of the virulent genotype. Using such plots, the time until the frequency of the virulence reaches a prescribed criterion, here defined as 0.9 , is found.

The added number of uninfected host growth days, $T_{\text {additional. }}$. Consider the same situation described in the previous section in which the resistant cultivar is introduced and the virulent genotype is in the genetic background of the pathogen population. In the long term, the system will stabilize at a new steady state (Table 2, bottom row). Figure 1C shows the dynamics of both pathogen genotypes and total density of uninfected crop. The shaded area under the curve is the added number of uninfected host growth days due to the use of the resistant crop before the virulent genotype has taken over, $T_{\text {additional }}$. After the virulent genotype has increased to its new steady state, the resistant cultivar will no longer have a positive effect on yield. $T_{\text {additional }}$ is calculated from

$$
T_{\text {additional }}=\int_{0}^{\infty}\left[H_{S}(t)-\bar{H}_{S}+H_{R}(t)-\bar{H}_{R}\right] d t
$$

where $\bar{H}_{S}$ and $\bar{H}_{R}$ are the steady-state densities of the susceptible and resistant cultivars after the system has converged to its new steady state.

\section{RESULTS}

Numerical results. The results of the numerical study are summarized in Figures 2 to 4 and Table 3.

Expected time until the virulent pathogen genotype invades,

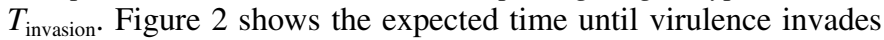
the pathogen population, $T_{\text {invasion, }}$ as a function of the cropping ratio, $\phi$. The invasion time is longer for both small and large cropping ratios than for intermediate values. The infection rate, $\beta$, has

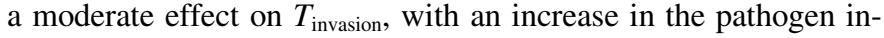
fection rate shortening the invasion time. At a cropping ratio of 0.5 , a fourfold decrease in infection rate results in an increase in the time until the virulent genotype invades in the population by $50 \%$. Immigration rate of the virulent pathogen genotype, $\delta_{2}$, has a much more profound effect on the expected time until the virulent genotype invades. This effect is largest at high cropping ratios. At a cropping ratio of 0.9 , a fourfold decrease in immigration results in an increase of more than $200 \%$ in the invasion time for the virulent form. The mutation rate from avirulent to virulent pathogen genotype also has a marked effect that is slightly more pronounced at smaller cropping ratios. At low mutation rates, the Ushape of the curve changes into a J-shape, with the shortest time for invasion occurring at large cropping ratios.

Time until the virulent genotype takes over, $T_{\text {take-over. }}$ Figure 3 shows the time until the virulent pathogen genotype has taken over the pathogen population, i.e., its frequency has increased beyond 0.9 , given that at time $(t)=0$ there is a small population of the virulent genotype in the genetic background of the pathogen population. The curves are all characteristically J-shaped with a monotonic decrease in $T_{\text {take-over }}$ with increasing cropping ratio. The 0.9 virulence frequency threshold is an arbitrary choice. The numerical value of $T_{\text {take-over }}$ decreases with decreasing threshold. We, however, have not found qualitative differences in the relation between $T_{\text {take-over }}$ and model parameters for lower values of the threshold. We therefore restrict the results to the 0.9 threshold throughout the paper. The effects of parameter values on this measure of durability are far less pronounced than for the waiting time until invasion (cf. Fig. 2). Changing the infection rate has a small effect (at $\phi=0.5$ ), with a fourfold increase shortening $T_{\text {take-over }}$ by $50 \%$. The immigration rate of the virulent pathogen genotype, $\delta_{2}$, hardly has any effect on the time until the virulent genotype takes over. This also holds for the initial density of the 
virulent pathogen genotype, $V(0)$, assuming that the initial population of the virulent strain in the pathogen population remains constant for total populations of different densities.

The added number of uninfected host growth days, $T_{\text {additional }}$. Changing the cropping ratio had very little effect on $T_{\text {additional }}$ in marked contrast to the two previous measures of durability, whereas changing the infection rate, $\beta$, of the pathogen had a great effect (Fig. 4). Note the difference in scales on the $y$ axis in Figure 4. At a cropping ratio of 0.9 , a fourfold decrease in the infection rate increased $T_{\text {additional }}$ by almost $500 \%$. The immigration rate of the virulent pathogen genotype has a small effect on the additional number of uninfected host growth days, which is most pronounced for small values of the cropping ratio. Again, in contrast to the previous analysis (Fig. 3), the initial density of the virulent genotype, $V(0)$, also influenced the amount of $T_{\text {additional }}$ (Fig. 4).

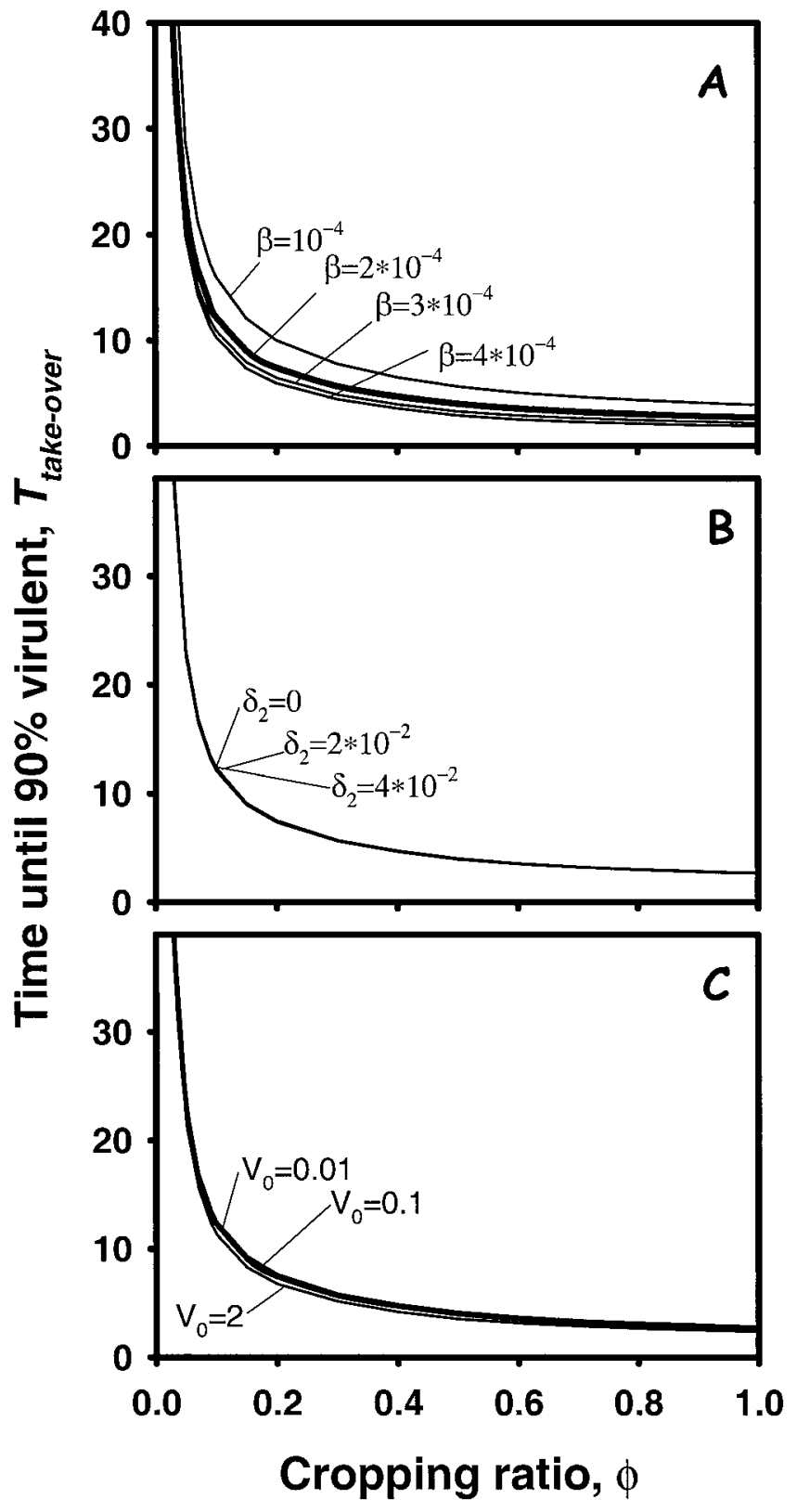

Fig. 3. Time until the virulent genotype comprises $90 \%$ of the pathogen population, given that it is initially present in the genetic background of the population, $T_{\text {take-over }}$, as a function of the cropping ratio, $\phi$ (cf. Fig. 1). A, The effect of infection rate, $\beta$. B, The effect of immigration rate of the virulent pathogen genotype, $\delta_{2}$. C, The effect of initial denst ity of the virulent genotype, $V_{0}$. Bold lines indicate the default parameter set $\beta=2 \times 10^{-4}, g=$ $200, \mu=3, \omega=0.1, \sigma=100, \delta_{1}=20, \delta_{2}=0.02, \varepsilon=10^{-5}, O=1$, and $V_{0}=0.1$.
Approximation formulas. In this section, we develop several approximations to the three measures of durability. In deriving such approximations, we make specific simplifying assumptions. Comparing the approximations with the results of the full model shows to what extent the measures of durability are sensitive to underlying processes. Results from the numerical analyses and the approximations are compared later.

Time until the virulent pathogen genotype invades, $T_{\text {invasion. }}$ After introduction of the resistant cultivar, the system converges to a new steady state (Fig. 1A). When the convergence to this new steady state is fast and the rates of mutation, $\varepsilon$, and immigration of the virulent genotype, $\delta_{2}$, are small, the system is far on its way toward convergence before the virulent genotype invades. In this situation, we can approximate the rate of appearance of new virulent pathogens by mutation and immigration, $K_{1}$, and the basic

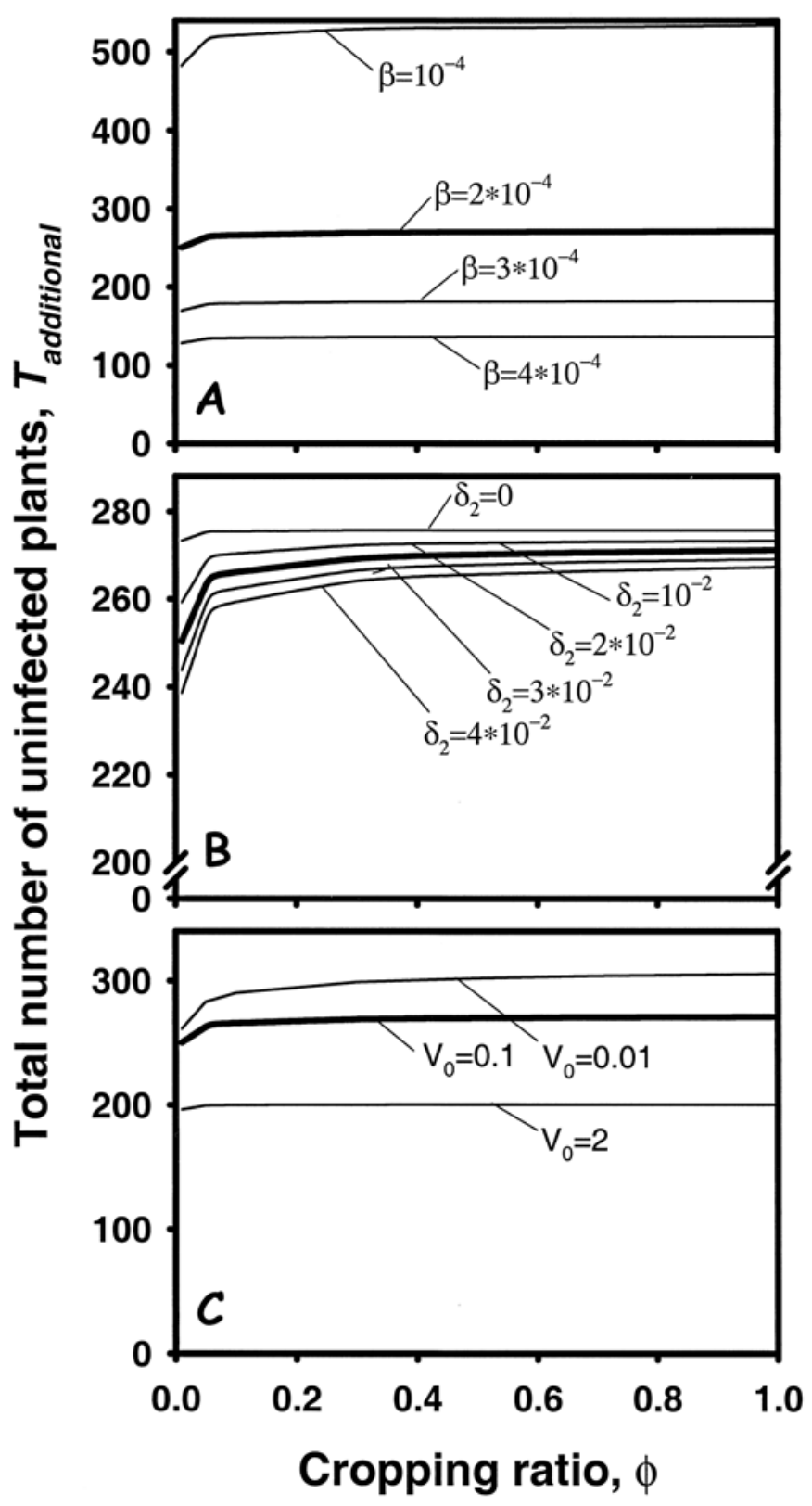

Fig. 4. Total number of additional uninfected crop growth days due to the release and deployment of the resistant cultivar, $T_{\text {additional, as a function of the }}$ cropping ratio, $\phi$. The quantity $T_{\text {additional }}$ is the hatched area in Figure 1, bottom graph. A, The effect of infection rate, $\beta$. B, The effect of immigration rate of the virulent pathogen genotype, $\delta_{2}$. $\mathbf{C}$, The effect of initial density of the virulent genotype, $V_{0}$. Bold lines indicate the default parameter set at $\beta=2 \times$ $10^{-4}, g=200, \mu=3, \omega=0.1, \sigma=100, \delta_{1}=20, \delta_{2}=0.02, \varepsilon=10^{-5}$, and $O=1$. 
reproductive number, $R_{0}^{\prime}$, with its value when the new steady state has developed. Substituting the appropriate steady-state values, we find

$$
\begin{gathered}
K_{1}=\varepsilon O\left(\frac{(1-\phi) \sigma g}{\mu}-\frac{\omega}{\beta}\right)+\delta_{2} O \\
R_{0}^{\prime}=\frac{\beta g}{\mu}\left(\frac{\mu}{\beta g}+\frac{\phi \sigma}{\omega}\right)
\end{gathered}
$$

Following the procedure to calculate $T_{\text {invasion }}$ outlined previously, the first approximation to $T_{\text {invasion, }}, \breve{T}_{1-\text { inv }}$, is simply the reciprocal of $\rho$, which finally yields

$$
1 / \breve{T}_{1-\text { inv }}=\left[\varepsilon O\left(\frac{(1-\phi) \sigma g}{\mu}-\frac{\omega}{\beta}\right)+\delta_{2} O\right]\left[1-\frac{1}{\frac{\beta g}{\mu}\left(\frac{\mu}{\beta g}+\frac{\phi \sigma}{\omega}\right)}\right]
$$

Figure 5 shows the simulated time until the virulent pathogen genotype invades together with approximation formula 12. For comparison, equation 12 is also plotted when there is no immigration $\left(\delta_{2}=0\right)$, from which the major dependence of this measure of durability on immigration becomes apparent. There is reasonably good agreement between the analytical approximation in equation 12 and the numerical solution over a wide range of cropping ratios, from 0.1 to 0.9 ; thus, for $\phi=0.2,0.4,0.6$, and 0.8 , the approximated value is $17,13,9$, and $6 \%$ smaller than the exact value, respectively. The difference between exact and approximated values is largest for small cropping ratios.

In the derivation of equation 12 , we assumed that all variables in the system were in their final steady state. We also assumed that the deviation is mainly due to the time it takes for the resistant cultivar after its release to reach its final level. Because the virulent pathogen genotype is not present yet, the resistant cultivar is not infected by any pathogen and the dynamics follow a simple monomolecular model

$$
H_{R}(t)=\frac{\phi \sigma}{\omega}\left(1-e^{-\omega t}\right)
$$

From this equation, we see that the steady-state assumption pertains to taking the limit of both $\omega$ and $\sigma$ to infinity such that $\sigma / \omega$ is a constant. In the second approximation, we assume that both parameters are large but not infinitely large. The calculations are straightforward if tedious and are given in the Appendix. For this second approximation, the time until the virulent pathogen genotype appears in the population, $\breve{T}_{2-\text { inv }}$, is given by

$$
\breve{T}_{2-\text { inv }}=\breve{T}_{1-\text { inv }}\left(1+\frac{1}{1+\frac{\beta g}{\mu} \frac{\phi \sigma}{\omega}} \frac{1}{1+\omega \breve{T}_{1-\text { inv }}}\right)
$$

where $\breve{T}_{1-\text { inv }}$ is the value derived as our first approximation (equation 12). This approximation gives a better description for smaller cropping ratios compared with $\breve{T}_{1-\text { inv }}$ (Fig. 5); the approximated values are now $10,8,6$, and $5 \%$ smaller than the exact value for cropping ratios of $0.2,0.4,0.6$, and 0.8 , respectively.

Time until the virulent genotype takes over, $T_{\text {take-over. The fre- }}$ quency of the virulent pathogen genotype in the population, $v(t)$, is given by $v(t)=V(t) /[A(t)+V(t)]$. Differentiation of $v$ with respect to time, substituting equations 2 and rearranging, finally yields the differential equation

$$
d v(t) / d t=\beta g H_{R}(t) v(t)[1-v(t)]
$$

The appearance of the density of the uninfected resistant cultivar, $H_{R}(t)$, in this equation implies that it is not possible to rewrite the model for increase of the virulence frequency given in equation 2 into a model of frequencies only, as is usual in population genetics. The dynamics of uninfected crop density, which is itself influenced by pathogen density, plays an essential role in the virulence dynamics of the system. Population genetics and population dynamics cannot therefore be considered independently.

If $H_{R}(t)$ is known, equation 15 can be solved. Denoting the initial frequency of the virulent pathogen genotype with $v(0)=v_{0}$, the solution is given by

$$
v(t)=\frac{1}{1+\frac{1-v_{0}}{v_{0}} \exp \left(-\beta g \int_{0}^{t} H_{R}(\sigma) d \sigma\right)}
$$

and a value for $T_{\text {take-over }}$ that corresponds to the virulent genotype reaching a given frequency, $v\left(T_{\text {take-over }}\right)=\theta$, is then found from

$$
\int_{0}^{T_{\text {take-over }}} H_{R}(t) d t=\frac{1}{\beta g} \ln \left[\left(\frac{\theta}{1-\theta}\right)\left(\frac{1-v_{0}}{v_{0}}\right)\right]
$$

The added number of uninfected host growth days, $T_{\text {additional }}$ It is possible to derive an explicit expression for $T_{\text {additional }}$ by neglecting the immigration of avirulent and virulent genotypes from outside the system. Recall from equation 10 that

$$
T_{\text {additional }}=\int_{0}^{\infty}\left[H_{S}(t)-\bar{H}_{S}+H_{R}(t)-\bar{H}_{R}\right] d t
$$

where $\bar{H}_{S}$ and $\bar{H}_{R}$ are the steady-state density of the susceptible

\begin{tabular}{|c|c|c|c|c|c|c|c|}
\hline $\begin{array}{l}\text { Measure } \\
\text { of durability }\end{array}$ & Criterion & $\begin{array}{l}\text { Pre-existing virulence } \\
\text { at sites of release }\end{array}$ & $\begin{array}{l}\text { Cropping } \\
\text { ratio, } \phi\end{array}$ & $\begin{array}{l}\text { Infection } \\
\text { rate, } \beta \\
\end{array}$ & $\begin{array}{l}\text { Immigration } \\
\text { rate, } \delta \\
\end{array}$ & $\begin{array}{l}\text { Mutation } \\
\text { rate, } \varepsilon \\
\end{array}$ & $\begin{array}{c}\text { Initial } \\
\text { density, } V_{0} \\
\end{array}$ \\
\hline$T_{\text {invasion }}$ & $\begin{array}{l}\text { Invasion (i.e., appearance and subsequent } \\
\text { establishment) of virulent strain }\end{array}$ & No & U-shaped & Moderate & Strong & Very strong & - \\
\hline
\end{tabular}
and resistant cultivars after the system has converged to its new steady state, for which

$$
\bar{H}_{S}+\bar{H}_{R}=\frac{\mu}{\beta g}
$$

The differential equation for $V(t)$ in equation 2 can be rewritten as

$$
\frac{1}{\beta g V(t)} \frac{d V(t)}{d t}=H_{S}(t)+H_{R}(t)-\frac{\mu}{\beta g}
$$

TABLE 3. Summary of the response of three measures of durability to changes in the cropping ratio and in epidemiological parameters 
Substituting equation 18 into 19 and the resulting formula into equation 10 , we find

$$
T_{\text {additional }}=\frac{1}{\beta g} \int_{0}^{\infty} \frac{1}{V(t)} \frac{d V(t)}{d t} d t=\frac{1}{\beta g}(\ln \{\bar{V}-\ln [V(0)]\})
$$

where $V$ is the steady-state value when the system has converged. Substituting this yields

$$
T_{\text {additional }}=\frac{1}{\beta g}\left[\ln \left(\frac{\sigma g}{\mu}-\frac{\omega}{\beta}\right)-\ln [V(0)]\right.
$$

\section{DISCUSSION}

In this discussion, we first tie up the results of the numerical study with the insight gained from the approximation formulas. Next, we discuss the model assumptions. We also discuss our results in the context of multiline crops and the consequences of the results for the regional deployment of resistant cultivars. For ease of reference, the principal results are summarized in Table 3.

Measures of durability. Expected time until the virulent pathogen genotype invades, $T_{\text {invasion. }}$. The U-shape of the $T_{\text {invasion }}$ versus $\phi$ curve (Fig. 2) is due to the balance of two processes that have to occur sequentially to result in invasion, the appearance of the virulent form and its subsequent establishment. The probability per unit time that a virulent genotype appears in the population through mutation is proportional to the number of avirulent genotype individuals, which in turn decreases with increasing cropping ratio. In the absence of immigration, the number of virulent individuals appearing by mutation in the population declines sharply at high cropping ratios, because few avirulent individuals are left to mutate into virulent genotypes. Figure 2 shows that immigration rate has a major effect on $T_{\text {invasion, especially at higher }}$ cropping ratios. This is understandable because, in this parameter region, the number of mutations is small because avirulent pathogen numbers are small, immigration then is the main source of virulent pathogen. The other process shows the opposite trend with cropping ratio; the probability of establishment increases with cropping ratio because more sites become available for sole colonization by the virulent form. The combination of these two trends explains the U-shaped curves in Figure 2. The approximation formulas (equations 12 and 14) and Figure 5 show that migration rate is a key parameter determining $T_{\text {invasion }}$. We conclude, therefore, that the immigration rate dominates the process of appearance of virulence at large cropping ratios. Figure 5 shows little difference between the numerical solutions of the full system and the steady-state approximation and the fast convergence approximation. This implies that $T_{\text {invasion }}$ is only to a minor extent influenced by the transient dynamics that occur after the introduction of the resistant cultivar. This is surprising because, in this transient phase, the density of the resistant cultivar increases from zero to its asymptotic level and the density of the avirulent strain decreases substantially. These changes do not influence the invasion of the virulent strain to any relevant extent.

Time until the virulent genotype takes over, $T_{\text {take-over }}$. Note again that our definition of this measure assumes that the virulent pathogen genotype is initially present at low densities in the genetic background of the population. At large cropping ratios, the amount of resource available to the virulent genotype is large compared with the avirulent form. The virulent form therefore increases rapidly. It takes less time for the virulent genotype to reach a frequency of 0.9 at large cropping ratios than at small cropping ratios, explaining the J-shape of the curves. The approximation formula (equation 17) and Figure 3 show that $T_{\text {take-over }}$ is rather insensitive to the initial frequency of the virulent genotype. For the default parameter set and an initial density of $V_{0}=0.1$, we calculate that $v_{0}=0.0000162$ and $\ln \left[\left(1-v_{0}\right) / v_{0}\right] \approx 11.0$. Increasing $V_{0}$ by a factor of 10 yields $\ln \left[\left(1-v_{0}\right) / v_{0}\right] \approx 13.3$. It is clear from Figure 3 that the dynamical behavior of $H_{R}(t)$ is such that this difference becomes even smaller in the exact (simulated) system.

The added number of uninfected host growth days, $T_{\text {additional }}$. The approximation formula (equation 21) shows that in the absence of pathogen immigration, the added number of uninfected growth days is independent of the cropping ratio. We interpret this surprising result according to the following biological mechanisms. When the cropping ratio is small, using the resistant cultivar does not give much additional yield. The virulent pathogen genotype however grows slowly only to a level such that the resistant cultivar hardly gives any extra yield compared with the susceptible cultivar. At large cropping ratios, the situation is reversed; the virulent genotype now increases very fast on the large amount of available host, rapidly canceling out the potential advantage of uninfected growth days from the release of the resistant cultivar. The yield gain due to the use of the resistant cultivar is however very large. The effect of cropping ratio on yield, and on the rate of increase of the virulent strain, results in $T_{\text {additional }}$ being independent of cropping ratio.

Comparing equation 21 and Figure 4 shows that the small decrease in $T_{\text {additional }}$ at smaller cropping ratios is due to immigration. Clearly, the effect of immigration diminishes when the initial density of the virulent genotype is large (Fig. 4C). Approximation equation 21 , furthermore, shows that the added number of healthy host growth days is logarithmically dependent on the density of the virulent pathogen genotype in the genetic background of the population.

Clearly, the three measures relate to different aspects of durability (Table 3 ). In particular, $T_{\text {take-over }}$ is derived for a system in which there is preexisting virulence at the time of release of the resistant cultivar, whereas $T_{\text {invasion }}$ is not. Can these be combined to measure the total time for the virulent form to take-over the population in the absence of preexisting virulence? Although this is possible by numerical simulation of equations 2 , simple summation of $T_{\text {invasion }}+T_{\text {take-over }}$ is not possible. The former is derived from the time to establishment but does not yield an explicit solution for the proportion of the virulent genotype in the population $[v(0)]$ for input into the calculation of $T_{\text {take-over }}$.

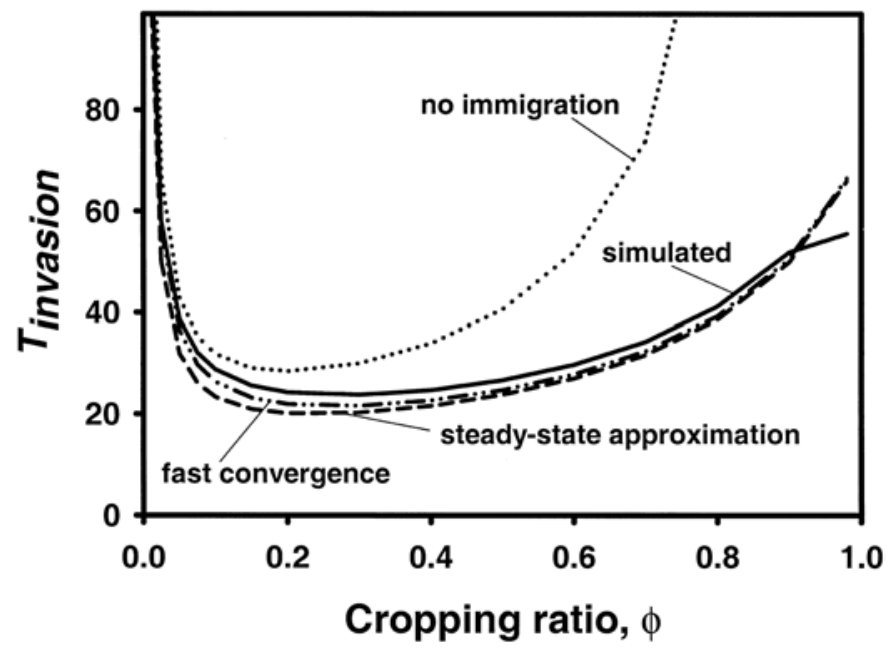

Fig. 5. Comparison of numerical solutions with the various approximation formulas to the measures of durability. Expected time until the virulent pathogen genotype invades, $T_{\text {invasion, }}$ is plotted as function of the cropping ratio, $\phi$. The line representing no immigration is the steady-state approximation when the immigration rate of the virulent pathogen genotype is zero. Parameter values are the default parameter set at $\beta=2 \times 10^{-4}, g=200, \mu=3$, $\omega=0.1, \sigma=100, \delta_{1}=20, \delta_{2}=0.02, \varepsilon=10^{-5}$, and $O=1$. 
Notes on the model assumptions. The model used in this paper is the simplest possible model that shows the effects of population dynamics and population genetics on durability. Moreover, in the analysis, we assumed the system to be in steady state at the moment the susceptible cultivar is released. This is a logical assumption given that we modeled a system with continuous planting and harvesting. In real life, plant-pathogen systems are hardly ever in steady state, not least because of the seasonal nature of planting and harvesting. Before generic conclusions can be drawn, the model has to be extended to include seasonal dynamics with pulsed (9) rather than continuous changes due to harvest and sowing and to include pathogen survival through the crop-free season. The model also ignores the effects of the spatial pattern of susceptible and resistant crop fields within a landscape $(30,31)$ and spatial differences in the field density between regions. This assumption is valid when the dispersal distance of the infectious units is much larger than the spatial scale of the crop-noncrop variations. For airborne pathogens, ignoring spatial structure will be a valid assumption when considering an assemblage of fields covering a region of a few square kilometers, whereas it will not be a valid assumption for soilborne pathogens on the same crop-noncrop spatial scale. We assumed that the pathogen does not experience a cost of resistance. There is, however, evidence that such costs of resistance exist (21) and in future work we have to take such possible costs into account. The models here are deterministic but stochastic effects are likely to play an important part in persistence of pathogen genotypes at low densities (10). Our simple model yields a set of baseline results, which show unexpected differences between measures of durability. These results can be used as a starting point in further study of the dynamics of virulent pathogen strains in the presence of a resistant cultivar. All of the model assumptions discussed will be considered in subsequent research in this project and will be returned to in future publications.

Multilines. The use of multilines and cultivar mixtures, in which each component carries one or more resistance gene unique to that component, is attracting renewed interest $(7,26,41)$ stimulated by increased ease of genetic transformation of cereals and other crops (34) and the demands for sustainability in disease control. Both theoretical and experimental studies on pathogen genotype dynamics in multilines and cultivar mixtures consider genotype frequency only $(20,22,23,39)$. The main conclusion of these studies is that the rate of increase in the frequency of the virulent genotype decreases with decreasing fraction of the resistant line in the crop, $\phi$. Our results show the same pattern. The time until the virulent genotype increases to high levels (0.9 in Fig. 3) decreases with increasing fraction of the resistant line in the crop. This would lead to the conclusion that the resistant cultivar should be used as little as possible to safeguard the source of resistance in order to prolong the durability of the resistant cultivar (33). Our study, however, shows that this conclusion relies on (i) the use of virulence frequency as a determinant of the usefulness of the resistant cultivar and (ii) on the assumption that the virulent genotype is already present in the pathogen population. If the virulent genotype is not present and the time until arrival by mutation or immigration and establishment is assumed to reflect durability, both a low and a high proportion of the resistant line in the crop prolong durability. Moreover, these analyses show that the benefit in additional uninfected host growth days is barely affected by the proportion of the resistant crop in the multiline or mixture.

Deployment strategies. The conventional approach to preserving the durability of newly released resistance genes is to introduce resistant cultivars at low cropping ratios (33). This reduces the selection pressure on the pathogen population and thus promotes durability. Deployment of resistance genes in crops exerts strong selection on the matching virulent pathogen genotype. Failure of resistance following the release and rapid expansion in the acreage of a newly resistant cultivar within a few years is well documented (1) and leads to the so-called boom and bust cycle as the domination of the virulent genotype renders the resistant cultivar useless. Our results show that low cropping ratio does indeed preserve durability (Figs. 2 and 3).

Nevertheless, our results challenge the universality of the buffering effect of low cropping ratios by showing that the expected time to invasion ( $T_{\text {invasion }}$ ) can be delayed by high as well as low cropping ratios. This holds when there is no preexisting virulence in the region of deployment of the resistant cultivars. In our model, yield gain of a resistant cultivar, measured by $T_{\text {additional, }}$, is only slightly dependent on cropping ratio. The effect, though small, is such that at low cropping ratios the yield advantage of the resistant cultivar is not fully exploited (Fig. 4). Yields are slightly larger at high cropping ratios in open systems with pathogen immigration (Fig. 4). Wide-scale empirical testing of the advantages of high cropping ratios for the release of resistant cultivars is premature. One possible alternative would be to use a high cropping ratio in a restricted part of the crop's area and none in the remaining area. If the total cropping area can be subdivided into several smaller areas with little migration between them, this might delay virulent genotype invasion and produce maximum added yields. Our model is not spatially explicit, except in allowing for immigration. Holt and Chancellor (11) have recently argued that to maximize area-wide strategic impact, small genotype units and random patterns were best, but to protect individual fields, large units and concentrated deployment were the best. These strategies require further investigation and will likely be reported in subsequent publications.

Final remarks. Besides the more detailed results presented in this paper, the principal message is that previous studies on the deployment of resistant cultivars and the dynamics of virulent genotypes are hampered by considering virulence frequency only. This has led to only one, and possibly not the most useful, definition of durability, namely the time until virulence take-over, $T_{\text {take-over }}$ As we have shown in this paper, other measures of durability such as the expected time until invasion of the virulent strain, $T_{\text {invasion }}$, and the additional yield, measured by the additional number of uninfected host growth days, $T_{\text {additional }}$, depend on the interplay between population dynamics and population genetics. We have shown that these interactions can have a major influence on the outcome of deployment of resistance studies.

\section{APPENDIX}

Derivation of equation 14. Substituting equation 9 into equation 6 , we can rewrite this expression as

$$
T=\int_{0}^{\infty} t \rho(t) e^{\int_{0}^{t} \rho(\sigma) d \sigma} d t=\int_{0}^{\infty} e^{t} \rho(\sigma) d \sigma d t
$$

Substitution of equation 12 into equation 5 and recalling that $\rho$ is the product of equations 3 and 4 , we find

$$
\rho(t)=\left[\varepsilon O\left(\frac{(1-\phi) \sigma g}{\mu}-\frac{\omega}{\beta}\right)+\delta_{2} O\left(1-\frac{1}{1+\frac{\beta g}{\mu} \frac{\phi \sigma}{\omega}\left(1-e^{-\omega t}\right)}\right)\right.
$$

Assuming that $\omega$ is large, we can approximate $\rho(t)$ by

$$
\rho(t) \approx \rho_{s t}\left(1-\frac{1}{1+\frac{\beta g}{\mu} \frac{\phi \sigma}{\omega}} e^{-\omega t}\right)
$$

where

$$
\rho_{s t}=\left[\varepsilon O\left(\frac{(1-\phi) \sigma g}{\mu}-\frac{\omega}{\beta}\right)+\delta_{2} O\right]\left(1-\frac{1}{1+\frac{\beta g}{\mu} \frac{\phi \sigma}{\omega}}\right)
$$


is the value of $\rho$ for the system in steady state. Substituting in equation $\mathrm{A} 1$, we find

$$
\begin{aligned}
& T=\int_{0}^{\infty} \exp \left(-\rho_{s t} t+\rho_{s t} \frac{1}{1+\frac{\beta g}{\mu} \frac{\phi \sigma}{\omega}} \int_{0}^{t} e^{-\omega \sigma} d \sigma\right) d t \approx \\
& \int_{0}^{\infty} e^{-\rho_{s t} t}\left(1+\rho_{s t} \frac{1}{1+\frac{\beta g}{\mu} \frac{\phi \sigma}{\omega}} \int_{0}^{t} e^{-\omega \sigma} d \sigma\right) d t=\frac{1}{\rho_{s t}}\left(1+\frac{1}{1+\frac{\beta g}{\mu} \frac{\phi \sigma}{\omega}} \frac{\rho_{s t}}{\rho_{s t}+\omega}\right)
\end{aligned}
$$

\section{ACKNOWLEDGMENTS}

The SECURE project is supported by the European Commission under the Fifth Framework Programme. Rothamsted Research is funded by the Biotechnology and Biological Research Council (BBSRC). C. A. Gilligan thanks BBSRC for support.

\section{LITERATURE CITED}

1. Bayles, R. A., Flath, K., Hovmøller, M. S., and de Vallavieille-Pope, C. 2000. Breakdown of the Yr17 resistance to yellow rust of wheat in northern Europe. Agronomie 20:805-811.

2. Bonhoeffer, S., Lipsitch, M., and Levin, B. R. 1997. Evaluating treatment protocols to prevent antibiotic resistance. Proc. Natl. Acad. Sci. USA 94:12106-12111.

3. Brown, J. K. M. 1995. Recombination and selection in populations of plant pathogens. Plant Pathol. 44:279-293.

4. Brown, J. K. M., and Wolfe, M. S. 1990. Structure and evolution of a population of Erysiphe graminis f. sp. hordei. Plant Pathol. 39:376-390.

5. Brun, H., Levivier, S., Somda, I., Ruer, D., Renard, M., and Chevre, A. M. 2000. A field method for evaluating the potential durability of new resistance sources: Application to the Leptosphaeria maculans-Brassica napus pathosystem. Phytopathology 90:961-966.

6. Feller, W. 1968. An Introduction to Probability Theory and Its Applications, vol. 1. 3rd ed. John Wiley \& Sons, New York.

7. Finckh, M. R., Gacek, E. S., Goyeau, H., Lannou, C., Merz, U., Mundt, C. C., Munk, L., Nadziak, J., Newton, A. C., de Vallavieille-Pope, C., and Wolfe, M. S. 2000. Cereal variety and species mixtures in practice, with emphasis on disease resistance. Agronomie 20:813-837.

8. Gale, J. S. 1990. Theoretical Population Genetics. Unwin Hyman, London.

9. Gubbins, S., and Gilligan, C. A. 1997. Persistence of host-parasite interactions in a disturbed environment. J. Theor. Biol. 188:241-258.

10. Gubbins, S., and Gilligan, C. A. 1999. Invasion thresholds for fungicide resistance: Deterministic and stochastic analyses. Proc. R. Soc. Lond. Ser. B. 266:2539-2549.

11. Holt, J., and Chancellor, T. C. B. 1999. Modelling the spatio-temporal deployment of resistant varieties to reduce the incidence of rice tungro disease in a dynamic cropping system. Plant Pathol. 48:453-461.

12. Hovmøller, M. S., Munk, L., and Ostergard, H. 1997. Modelling virulence dynamics of airborne plant pathogens in relation to selection by host resistance in agricultural crops. Pages 173-190 in: The Gene-forGene Relationship in Plant Parasite Interactions. I. R. Crute, E. B. Holub, and J. J. Burdon, eds. CABI, Wallingford, UK.

13. Johnson, R. 1978. Practical breeding for durable resistance to rust diseases in self-pollinating cereals. Euphytica 27:529-540.

14. Johnson, R. 1979. The concept of durable resistance. Phytopathology 69:198-199.

15. Johnson, R. 1981. Durable resistance-Definition of genetic control, and attainment in plant breeding. Phytopathology 71:567-568.

16. Johnson, R. 1984. A critical analysis of durable resistance. Annu. Rev. Phytopathol. 22:309-330.
17. Johnson, R. 1992. Reflections of a plant pathologist on breeding for disease resistance, with emphasis on yellow rust and eyespot of wheat. Plant Pathol. 41:239-254.

18. Kiyosawa, S. 1982. Genetics and epidemiological modeling of breakdown of plant-disease resistance. Annu. Rev. Phytopathol. 20:93-117.

19. Kolmer, J. A. 1996. Genetics of resistance to wheat leaf rust. Annu. Rev. Phytopathol. 34:435-455.

20. Lannou, C., and Mundt, C. C. 1996. Evolution of a pathogen population in host mixtures: Simple race-complex race competition. Plant Pathol. 45:440-453.

21. Leach, J. E., Cruz, C. M. V., Bai, J. F., and Leung, H. 2001. Pathogen fitness penalty as a predictor of durability of disease resistance genes. Annu. Rev. Phytopathol. 39:187-224.

22. Leonard, K. J. 1977. Selection pressures and plant pathogens. Ann. N.Y. Acad. Sci. 287:207-222.

23. Marshall, D. R., and Weir, B. S. 1985. Multiline varieties and disease control. 5. The dirty crop approach with complex-mixtures of genotypes based on overlapping gene sets. Theor. Appl. Genet. 69:463-474.

24. Metz, J. A. 1978. The epidemic in a closed population with all susceptibles equally vulnerable: Some results for large susceptible populations and small initial infections. Acta Biotheor. 27:75-123.

25. Østergård, H. 1987. Estimating relative fitness in asexually reproducing plant pathogen populations. Theor. Appl. Genet. 74:87-94.

26. Østergård, H. 1987. Predicting development of epidemics in cultivar mixtures. Phytopathology 73:166-172.

27. Østergård, H., and Hovmoller, M. S. 1991. Gametic disequilibria between virulence genes in barley powdery mildew populations in relation to selection and recombination. 1. Models. Plant Pathol. 40:166-177.

28. Paillard, S., Goldringer, I., Enjalbert, J., Doussinault, G., de VallavieillePope, C., and Brabant, P. 2000. Evolution of resistance against powdery mildew in winter wheat populations conducted under dynamic management. I. Is specific seedling resistance selected? Theor. Appl. Genet. 101:449-456.

29. Paillard, S., Goldringer, I., Enjalbert, J., Trottet, M., David, J., de Vallavieille-Pope, C., and Brabant, P. 2000. Evolution of resistance against powdery mildew in winter wheat populations conducted under dynamic management. II. Adult plant resistance. Theor. Appl. Genet. 101:457-462.

30. Park, A. W., Gubbins, S., and Gilligan, C. A. 2001. Invasion and persistence of disease in a spatially structured metapopulation. Oikos 94:162-174.

31. Park, A. W., Gubbins, S., and Gilligan, C. A. 2002. Extinction times for spatially-structured closed epidemics. Ecol. Lett. 5:747-755.

32. Parlevliet, J. E. 2002. Durability of resistance against fungal, bacterial and viral pathogens: Present situation. Euphytica 124:147-156.

33. Pink, D., and Puddephat, I. 1999. Deployment of disease resistance genes by plant transformation-A 'mix and match' approach. Trends Plant Sci. 4:71-75.

34. Stuiver, M. H., and Custers, J. H. H. V. 2001. Engineering disease resistance in plants. Nature 411:865-868.

35. van Herwaarden, O. A., and Grasman, J. 1995. Stochastic epidemicsMajor outbreaks and the duration of the endemic period. J. Math. Biol. 33:581-601.

36. Vanderplank, J. E. 1984. Disease Resistance in Plants. 2nd ed. Academic Press, New York.

37. Vera Cruz, C. M., Bai, J., Ona, I., Leung, H., Nelson, R. J., Mew, T.-W., and Leach, J. E. 2000. Predicting durability of a disease resistance gene based on an assessment of the fitness loss and epidemiological consequences of avirulence gene mutation. Proc. Natl. Acad. Sci. USA 97:13500-13505.

38. Williamson, M. 1996. Biological Invasions. Chapman \& Hall, London.

39. Wolfe, M. S., and Barrett, J. A. 1977. Population genetics of powdery mildew epidemics. Ann. N.Y. Acad. Sci. 287:151-163.

40. Zeigler, R. S., Teng, P. S., and Leong, S. A. 1994. Rice Blast Diseases. CABI Biosciences, Wallingford, UK.

41. Zhu, Y. Y., Chen, H. R., Fan, J. H., Wang, Y. Y., Li, Y., Chen, J. B., Fan, J. X., Yang, S. S., Hu, L. P., Leung, H., Mew, T. W., Teng, P. S., Wang, Z. H., and Mundt, C. C. 2000. Genetic diversity and disease control in rice. Nature 406:718-722. 